\title{
Advances in Multi-Sensor Systems for In Situ Remote Sensing of Australian Forest Canopy Processes
}

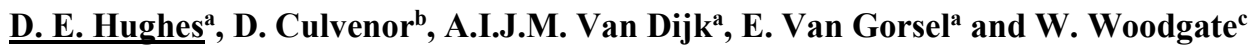 \\ ${ }^{a}$ Water and Landscape Dynamics, Fenner School of Environment and Society, The Australian National \\ University, Australian Capital Territory. ${ }^{b}$ Environmental Sensing Systems, Melbourne, Victoria. ${ }^{c}$ CSIRO \\ Land and Water, Canberra, Australian Capital Territory \\ Email:dale.hughes@anu.edu.au
}

\begin{abstract}
Remote sensing has been the mainstay of much environmental research for many years. Data acquired from remote sensing systems deployed on spacecraft and aircraft have provided insight into environmental processes that are difficult or impossible to obtain otherwise. However, traditional remote sensing systems are expensive, highly specialised and may lack the ability to be moved or quickly deployed.
\end{abstract}

Fortunately, the cost of sensors like those used in existing remote sensing systems has decreased over time, and their availability and capability has increased. It is now possible to build and deploy ground-based remote sensing systems with capabilities similar to those on traditional platforms. In this context we define 'in situ remote sensing systems' to be within, above, or otherwise adjacent to the vegetation under study but not in direct contact with it. Such systems typically have a measurement range of a few tens of metres, can be deployed quickly and easily moved to new areas of study when required.

This paper describes the development of in situ remote sensing systems as has occurred in Australia. The paper also outlines some of the challenges that were met during development and use of several generations of in situ remote sensing systems and presents contemporary work being done for the next generation of systems that will further expand our scientific measurement capability.

Initial development of in situ remote sensing systems started in about 2003 with the deployment of the first single pixel multi-angle spectrometer at the CSIRO Tumbarumba field site in south-east New South Wales. Though this was a basic system, it provided much useful data, both in a scientific sense as well as providing information that would be useful in engineering the next generation of sensor systems. Early work in comparing acquired in situ data to satellite sensed data, and radiative modelling was complicated; difficulties included the effects of a non-homogenous forest canopy and the angular distribution of leaves.

In 2013 a much more sophisticated system capable of hyperspectral and thermal imaging was installed at the same site, and this system continues to provide detailed and calibrated spectral and thermal time series images of forest canopy dynamics. The ability to examine specific and more uniform regions of interest within the forest canopy is the real value of imaging systems, as opposed to single footprint area-averaged measurements. Because of the high spatial resolution of the acquired data, it is now possible to examine 'within-tree' variability as well as 'between-tree' variability. The system provides much data for scientific analysis, and points to some of the engineering and data handling issues that influence the way future systems are developed.

Building upon these earlier systems, the Australian National University has embarked on a project to build a 'state of the art' sensor system that will extend the range of observed wavelengths and be deployable on various platforms for either short-term or extended observation campaigns. This new system uses a variety of sensors that cover the visible, short- and long-wave infrared wavelengths. In the first instance, scientific applications will focus on in situ remote sensing at the ANU Forest Research Facility at the National Arboretum in Canberra.

Some important lessons have been learnt over the past 15 years. In situ remote sensing systems require careful design and engineering if they are to reach their full potential. Instrument calibration, handling the vast amount of acquired data and efficient data reduction are significant factors in the successful use of such systems. This contribution concludes with a speculative view of the future direction of science infrastructure in this field.

Keywords: Hyperspectral, thermal, LIDAR, infrared, remote sensing, forest 


\section{INTRODUCTION}

A variety of remote sensing systems have been developed as researchers push the boundaries of what can be measured by traditional methods. Research questions cover a wide range of scales; from leaf level, to plot, to national, then continental and ultimately through to a global scope. As the scope grew traditional methods of acquiring data have become difficult or impossible because of cost or access. Also, interest in integrating over scales through increasingly detailed models requires data streams from various scales to constrain those models. These factors have led to systems that can infer ecological processes from non-contact measurements made from remote instruments on spacecraft or aircraft. By recording suitably calibrated spectral signatures of the vegetation under investigation, it has become possible to infer what processes are occurring within the vegetation under study more or less in real-time.

While it is still necessary to collect samples from representative trees, it has become possible to examine a plot, or even an entire forest, and to do so for an extended period. The developments that have made this approach possible are the subject of this contribution. We look at developments of remote sensing techniques that may be used to fill the gap between, on the one hand, physical collection of measurements at leaf scale and, on the other, measurements made by aircraft or from space.

We describe observations at this intermediate scale as 'in situ remote sensing'. We accept the now common usage of the term 'remote' to refer to non-contact forms of sensing rather than a necessarily large distance between sensor and target (as in e.g., 'TV remote control'). 'In situ' here refers to using sensors on a tower, vehicle or another platform that is within a few tens of metres of the vegetation under study. The advantage of in situ remote sensing systems is that they can still cover relatively large areas, are accessible, do not require the operation of expensive platforms, and can be quickly deployed and recovered as circumstances require.

\section{EARLY DEVELOPMENTS}

To our knowledge, the first Australian tower-mounted in situ hyperspectral remote sensing system was developed and deployed by the CSIRO at its Tumbarumba field site in 2003 as described by Leuning et al. (2006). During the several years that the system operated much data was acquired, and useful experience was gained in building and deploying in situ remote sensing systems. The system used a single footprint (pixel) sensor that covered the spectral range of 300-1150 nm with 256 wavelength bins and the instrument measured the average reflected radiance in its field of view every daylight hour. The sensor input could be either the incident irradiance or the reflected radiances at four azimuth angles. To assess system noise, measurements of sensor dark current were also made.

Calibration of the device was undertaken on clear days with a Spectralon panel of known reflectance at solar noon. This allowed characterization of the sensor, optical fibre, mirror assemblies and cosine-corrected hemispherical diffuser. Once the instrument was properly calibrated calculating the reflectance of the forest canopy under investigation was straightforward.

An important advantage of the system was that the optical paths for both the incident irradiance and reflected radiance were very similar: a mirror was used to switch between inputs and this technique resulted in reliable measurements and a very stable instrument calibration. Over time various mirror configurations were installed to change the instrument field of view. As originally deployed at the top of a $70 \mathrm{~m}$ tower the sensor measurement footprint was approximately $7 \mathrm{~m}$ by $3 \mathrm{~m}$; this was later increased to approximately $19 \mathrm{~m}$ by $8 \mathrm{~m}$ to reduce the effect of leaf angle distribution on the reflectance measurements.

Over time, however, several disadvantages of the system became apparent:

- the limited number of observation azimuth angles,

- the fixed zenith angle view of the forest canopy,

- most importantly, the single pixel nature of the measurement.

Early work in comparing acquired in situ data to satellite sensed data and radiative models was complicated for a variety of reasons. The effects of a non-homogenous forest canopy and the angular distribution of leaves being significant difficulties. To have a picture would be so much better!

\section{MORE RECENT DEVELOPMENTS}

Hyperspectral sensor technology has advanced considerably since the first instrument was devised and it is now possible to purchase hyperspectral line-scan cameras with high spatial and spectral resolution along with adequate sensitivity and stability, at relatively low cost. Such sensors are used routinely on aircraft and 
spacecraft and are known as 'push-broom' systems, as the sensor is moved to generate a two-dimensional image, similar to a flatbed scanner. Over the same period, much work has been done to reduce the size and cost of Long-Wave Infra-Red (LWIR) cameras, and sensitive and stable LWIR cameras are now readily available.

By positioning a line-scan camera on a moving platform that is fixed to a tower, it is possible to acquire hyperspectral and thermal images of the vegetation of interest. Provided the instruments are suitably calibrated radiance and reflectance images at a variety of wavelengths can be generated. Reflectance images or derived data (e.g., one of the various vegetation indices) can be generated as often as required, wherever required and covering a range of scales from leaf to tree to plot, by choosing the platform and height and by adjusting the optics of the camera. A range of ecophysiological processes can now be observed without having to be very close to the vegetation.

Work on just such an instrument started in 2012 and the first version of the system was deployed at the CSIRO Tumbarumba field site in mid-2013. This made it possible to acquire high-quality hyperspectral and thermal images simultaneously in real-time (van Gorsel et al. 2015). The final iteration of the system (as of 2016) included a Headwall Photonic hyperspectral camera with approximately 700 wavelength bins covering the spectral range 400-800 nm, a FLIR A655sc LWIR camera covering the 7-14 $\mu \mathrm{m}$ range, an all-sky camera to assess the sky conditions (especially, whether there was cloud cover), and an Ocean Optics spectrometer to measure incident irradiance.

At an approximate distance to target of $60 \mathrm{~m}$, image resolution is sufficient to discern small branches and clumps of leaves. This facilitates important analysis of both within-tree and between-tree variability. Some sample data of this type is shown in Figures 1 and 2 below.

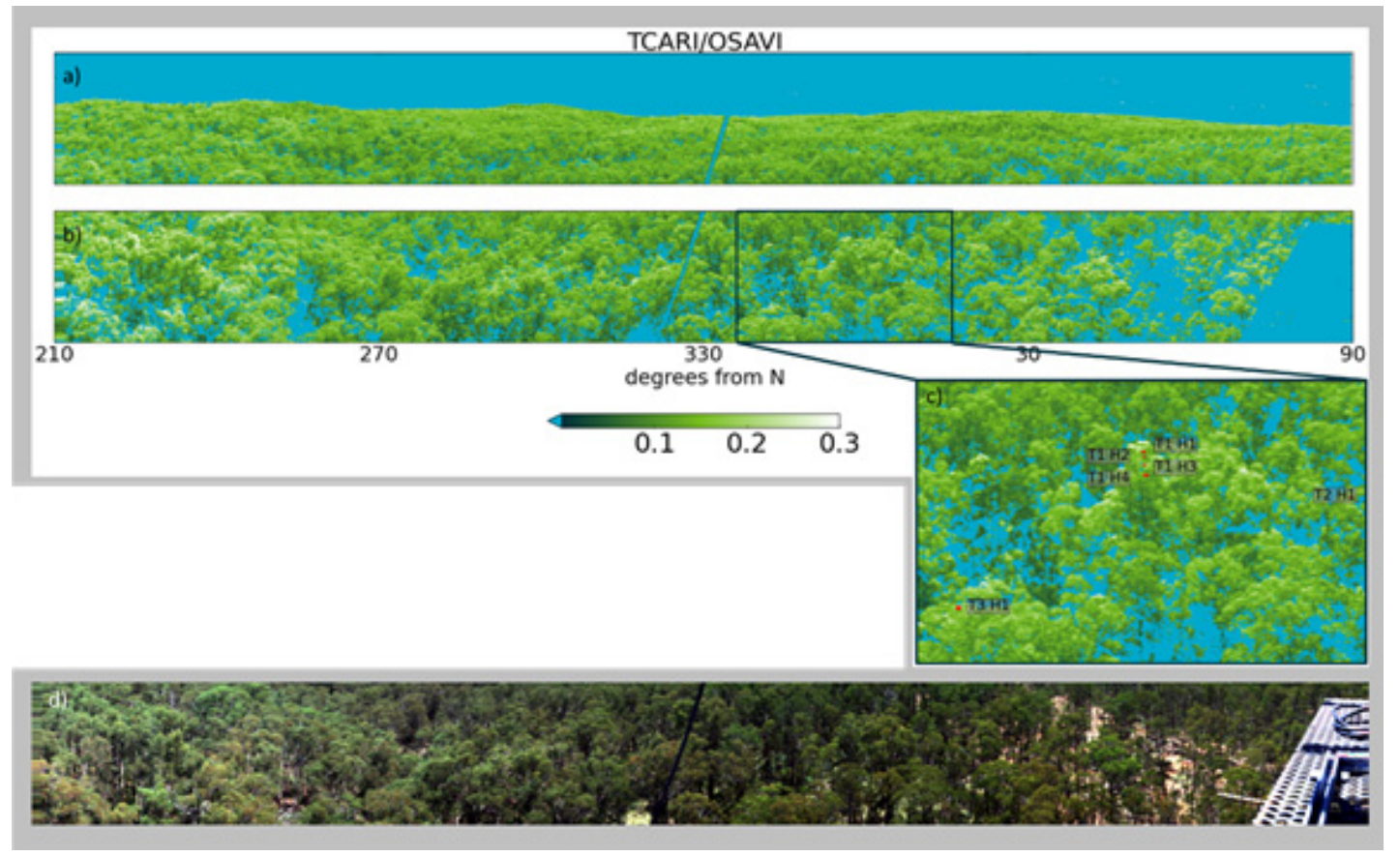

Figure 1. A sample hyperspectral image presented as a vegetation index (upper) and a true colour image (lower) generated from the recorded hyperspectral data (from van Gorsel et al., 2015) TCARI/OSAVI is the Transformed Chlorophyll Absorption in Reflectance Index/Optimized Soil-Adjusted Vegetation Index (TCARI/OSAVI) . Example within-tree and between-tree data from selected trees is shown in Figure 2. 

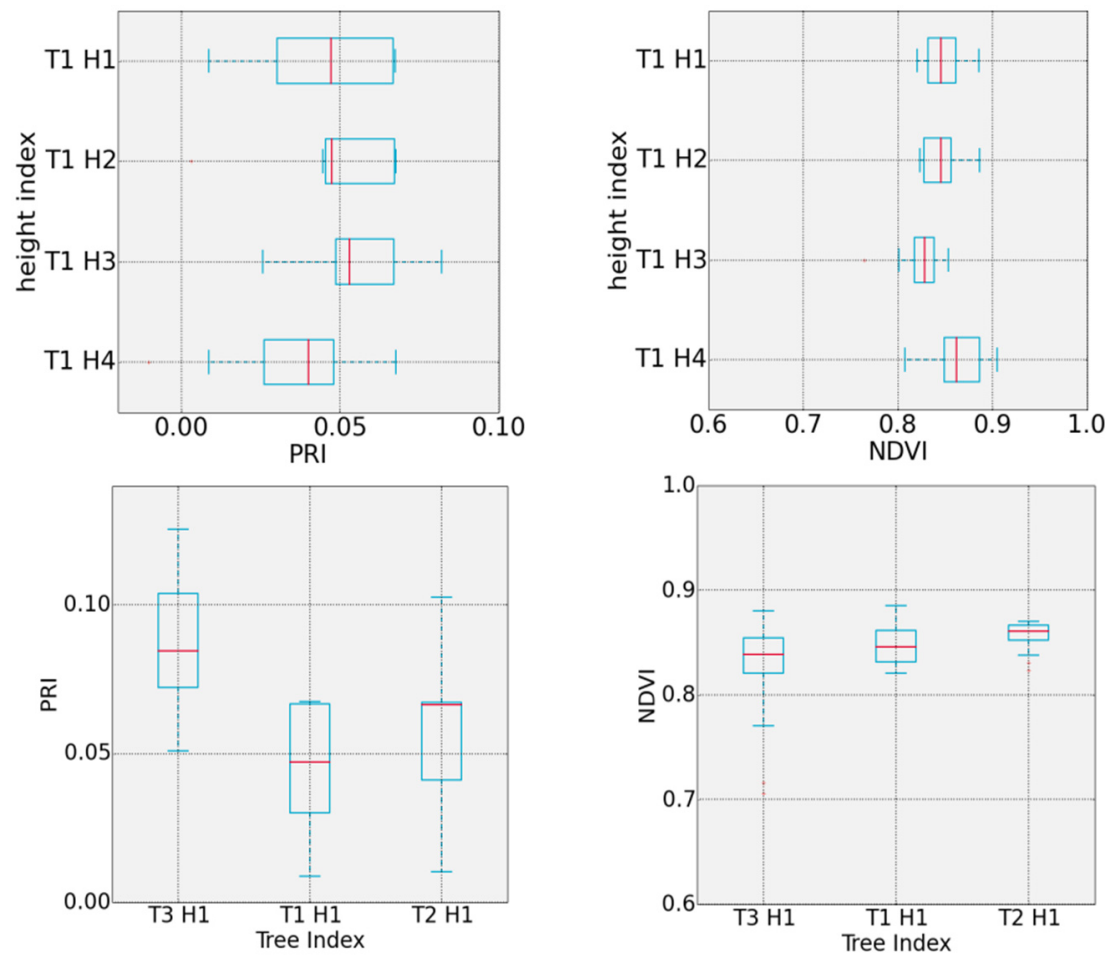

Figure 2. Examples of within-tree variability (upper row) and between-tree variability (lower row) for selected vegetation indices extracted from the images shown in Figure 1. PRI is the Photochemical Reflective Index and NDVI is the Normalised Difference Vegetation Index. Further work is required to determine if the differences are due to biophysical effects or due to structural effects (from van Gorsel et al., 2015)

Concerning LWIR measurements, Aubrecht et al. (2016) showed that a number of ancillary data streams and post-processing steps are required for accurate inference of canopy temperature from thermal cameras. Relative humidity, distance to target and sky temperate data are needed to properly account for environmental thermal interferences between the sensor and target. Image post-processing is required to correct for the atmospheric transmission when target range varies substantially within each thermal image. This step also requires dataset co-registration, which can be challenging because of movement of the sensor system and target canopy over time. Additionally, specific sensors may be subject to image artefacts which require additional calibration or post-processing. For example, Figure 3 shows that the FLIR A655sc can suffer from a step-change in temperature output under dynamic ambient temperature conditions due to the on-board non-uniformity correction.

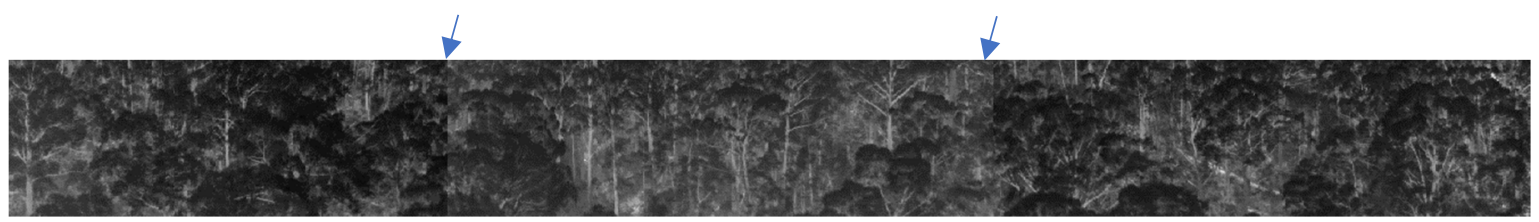

Figure 3. A panorama thermal image of the forest canopy. This image shows two of the LWIR sensor artefacts that have caused some difficulty in data analysis as noted in the text. The step changes in the image brightness (at arrows) are caused by the automatic image adjustment made by the camera.

Experience has shown that the two most useful operating modes are data acquisition at predefined time intervals, or at predefined sun angles. In the latter 'sun synchronous' mode, the defined sun angles were taken to be solar noon (i.e. the time at which the sun was at its highest elevation for the day) and when the sun was at the same elevation as its maximum elevation on the winter solstice, both before and after solar noon. Except for mid-winter day, these three sun angles occur every day of the year but their exact timing changes each day. 
The operating software calculates accurate solar position coordinates each day to determine when to perform an image acquisition scan.

Some lessons were learnt from the development of this system;

- While data acquisition every hour produced some interesting insights, the resulting hundreds of gigabytes of daily data was a challenge to store and process.

- In-built data compression, at the cost of a reduction in image detail, may be required to reduce dataset size before storage to increase temporal resolution.

- Radiometric and wavelength calibration of the irradiance and hyperspectral sensors requires great care, especially because of the partial overlap between sensor ranges and the available calibration sources.

- Repeated instrument validation/calibration is required to account for instrument drift.

- Radiometric values acquired by the LWIR camera can be difficult to convert into accurate temperatures under certain acquisition conditions even if the necessary corrections for ambient temperature, relative humidity and target range are applied.

Despite these challenges, the system has proved to be a valuable asset and has acquired a relatively long time series of spectral and thermal dynamics of the forest canopy and this information can be linked to real-time measurements of carbon and water fluxes that are also acquired at the site.

\section{CURRENT DEVELOPMENTS}

Progress in sensor development has continued and hyperspectral and LWIR cameras have been further miniaturised. Relatively low-cost (albeit broad-band) Short-Wave Infra-Red (SWIR) line-scan cameras can now be purchased, extending routinely observable wavelengths into spectral regions previously considered prohibitively expensive.

The Forest Spectroscope developed in 2017 at the Australian National University (ANU) integrates hyperspectral, SWIR, LWIR and all-sky cameras along with ancillary instruments into a single, compact sensing system that can be mounted on a tower, elevated work platform or vehicle. The spectral range of the combined instruments covers the $400-800 \mathrm{~nm}, 800-1700 \mathrm{~nm}$ and $7-14 \mu \mathrm{m}$ bands with slightly varying spectral and spatial resolution in the various bands because of the individual instrument characteristics. A LIDAR operating at $905 \mathrm{~nm}$ with a $120 \mathrm{~m}$ range and beam divergence of $0.2^{\circ}$ is also integrated into the system. In addition to giving target range data, the LIDAR will also provide details of the target canopy or tree structure.

The system can be used as an in situ remote sensing system for studying the spectral characteristics and dynamics of target vegetation. It can be deployed for short periods or can be left in situ for an extended period of time while operating using solar power.

The first area for investigations will be the ANU forest research facility at the National Arboretum in Canberra. The ANU forest research facility is intensively instrumented with a distributed network of weather, soil and tree growth sensors. The data collected with the Forest Spectroscope can help answer questions about how micro-scale variation in climate and extreme weather events affect the growth of trees as they mature from individual trees to mature forest ecosystems, and how this differs between and within species.

Furthermore, the Arboretum at large provides a unique opportunity to characterize entire stands of different tree species. The Arboretum is home to over 48,000 trees growing in 94 forests across the 250 hectare site, most of them in single-species stands. While many of the forests are relatively young, there are also mature pine and 100 year-old Himalayan Cedar and Cork Oak forests. Tree species include Australian as well as foreign species, of widely varying phenology and leaf functional traits. In this context, the sensing system is expected to provide dense remote sensing observations at different scales so that these insights can be scaled from individual trees to forests to the globe. It will also allow the use of the diverse forests as a satellite 'colour checker' for 'ground-truthing' as part of mission calibration and validation activities and radiative transfer model development.

The ultimate aim is to develop an automated and integrated sensing system that can acquire high-quality hyperspectral and thermal images on demand for an extended period of time. The availability of this, and, similar systems will provide new and interesting science opportunities. 


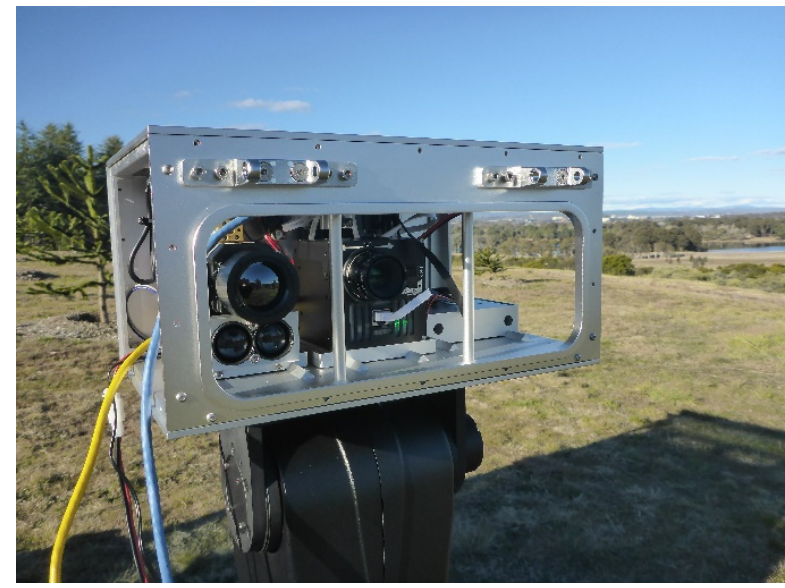

Figure 4. The ANU forest spectroscope in the first operational field trials in August 2017.

Note that external casing is removed here and that the SWIR camera was not installed.

\section{ENGINEERING CHALLENGES}

Development of the systems described above has meant that many technical problems have had to be addressed over time. Each iteration has built upon earlier versions making the remote sensing systems more capable, reliable and easier to use. Despite the advances and improvements in sensor technology some challenges remain:

- Calibration of optical sensors, both for spectral wavelength and light intensity measurements, needs to be done carefully so that accurate and reproducible reflectance measurements can be made at the required wavelengths. Some optical features are narrow-band and require precise wavelength calibrations, while accurate light intensity calibration is required for both the incident irradiance and reflected radiance measurements. Errors or changes in the instrument calibration will introduce significant errors in the calculated reflectance values or vegetation indices.

- Calibration of thermal images remains a challenge due to issues with LWIR camera design and environmental effects on images. Measurement of target range, air temperature and relative humidity and an understanding of the instruments characteristics is required to fully correct and convert the radiometric measurements into accurate temperature measurements.

- Data handling is an issue because of the large amount of data acquired by the image sensors. The acquisition of short interval time series can rapidly accumulate a very large amount of data to store, transfer and quality control. Suitable tools need to be developed to quickly reduce the raw image files to images showing reflectance or vegetation indices.

- Removing the effect of the angular distribution of leaves (bi-directional leaf reflectance or BDRF) remains challenging and a completely satisfactory solution is yet to be found. Oversampling the scene through a wide range of angles and sun-synchronous times and spherical scanning using a design like the Forest Spectroscope should help to resolve the issue, but more work needs to be done.

\section{THE FUTURE}

Sensor technology continues to evolve and new types of hyperspectral sensors that instantaneously produce two-dimensional images are being brought to the market. Also, a number of low-cost SWIR and LWIR cameras are available, and in the near future this may create new research opportunities where this type of technology can acquire data that was previously unobtainable.

To speculate: the convergence of the Internet-Of-Things, Big Data and ever expanding network connectivity as well as the decreasing cost of high performance sensors and computer systems will mean that high quality spectral and thermal data can be acquired at relatively low cost at multiple sites and potentially be available in near real-time. Machine learning systems and artificial intelligence software may be useful tools for analyzing and interpreting the large amount of image data made available by in situ remote sensing systems. Libraries of spectral signatures could be developed and used to automatically determine forest health, productivity or other factors of interest. The issue of device and system calibration and stability appear to be the only remaining limiting factors to the vast expansion of observational capability that is possible. The ongoing increase in data 
acquisition, processing, transfer and storage is likely to continue to push the boundary of the temporal, spatial and spectral resolution and extent that can practically be achieved.

\section{CONCLUSION}

An overview of the advancement of known in situ remote sensing systems in Australia has been provided. Sensor technology has changed significantly since the first simple system was devised 15 years ago and advanced imaging systems covering a wide range of scientifically interesting wavelengths are now available. We can simultaneously collect co-incident images from the visible wavelengths through to the invisible LWIR wavelengths with high spectral, temporal and spatial resolution. The spatial scale of such images extends from sizes not much greater than a leaf, to tree canopies up to plot sizes and such images can be recorded as often as needed to obtain the required time series. The ability to collect other synchronized biophysical data (e.g. carbon and water fluxes) along with imagery data allows us to further improve our understanding of the linkages between biophysical processes and optical and thermal responses.

\section{REFERENCES}

Aubrecht, D.M., B.R. Helliker, M.L. Goulden, D.A. Roberts, C.J. Still and A.D. Richardson (2016). Continuous, long-term, high-frequency thermal imaging of vegetation: Uncertainties and recommended best practices. Agricultural and Forest Meteorology, 228-229, 315-326. doi: http://dx.doi.org/10.1016/j.agrformet.2016.07.017

Leuning, R., D. Hughes, P. Daniel, N. Coops and G. Newnham (2006). A multi-angle spectrometer for automatic measurement of plant canopy reflectance spectra. Remote Sensing of Environment, 103, 236 - 245

Van Gorsel, E., D. Hughes and A. Cabello (2015). The hyper phenocam (thermal hyperspectral phenology camera system). Paper presented at the 9th EARSeL SIG Imaging Spectroscopy workshop, Luxembourg, April 14-16. 